\title{
Organocatalytic Synthesis of Chromans and Benzopyrano[3,4-c]pyrrolidine
}

Gategory

Synthesis of

Heterocycles

Key words

benzopyrano-

[3,4-c]pyrrolidine

oxa-Michael

reaction

domino reaction

organocatalysis

chroman
Significance: Reported is the enantioselective synthesis of trans-disubstituted chromans through the use of a sequential oxa-MichaelMichael reaction sequence and the subsequent synthesis of benzopyrano[3,4-c]pyrrolidine via reductive amination and $\mathrm{N}$-alkylation. trans-Disubstituted chromans were obtained in 62-91\% isolated yield with diastereomeric ratios ranging from 94:6 to 97:3 and 93-98\% ee. The reaction conditions are quite mild and the success of the reaction in either toluene or chloroform allows for a wider solubility range of the phenol starting material. Although the use of many pyrrolidine-containing organocatalysts resulted in product formation, (2R)-2-\{diphenyl[(trimethylsilyl)oxy]methyl\}-pyrrolidine gave the highest product yield. A limitation was the failure of an electron-rich methoxy-substituted phenol to provide the corresponding chroman.
Comment: Benzopyrano[3,4-c]pyrrolidines have been investigated in a wide variety of therapeutic areas, including anti-psychotics or anti-depressants (Lavielle et al. EP691243 A1, 1996), Alzheimer's disease (Asberom et al. WO2007084595 A2, 2007), obesity, insulin resistance, hypertension (Yao et al. WO2006002349 A1, 2006), and benign prostatic hyperplasia (A. R. Haight et al. Org. Process Res. Dev. 2004, 8, 897). Given this variety of therapeutic applications, this new method should garner a fair amount of interest from the drug discovery community. This method appears to be quite suitable for process-scale chemistry, due to its mild conditions and synthetic step reduction when compared to more common techniques, such as the one described by Dubuffet and coworkers (Bioorg. Med. Chem. Lett., 1999, 9, 2059). This method also circumvents the use of chiral column chromatography, accelerating the drug discovery process even further. 